\title{
ARTÍCULOS
}

\section{PROPOSAL OF USING SCALING FOR CALCULATING FIELD-NORMALIZED CITATION SCORES}

\author{
Propuesta de utilizar escalado para calcular la \\ citación normalizada por disciplina
}

\section{Robin Haunschild and Lutz Bornmann}
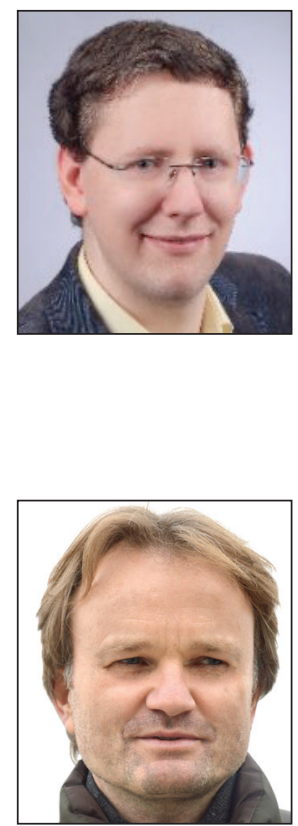

Robin Haunschild studied chemistry in Hannover and Marburg and received a scholarship from the German Research Foundation which he used to stay at Rice University in Houston, Texas. Afterwards, he has spent four years at the Karlsruhe Institute of Technology. In total, he worked as a theoretical chemist for nearly 10 years. In 2014, he joined the Max Planck Institute of Solid State Research in Stuttgart (Germany). His main research activities include bibliometrics and altmetrics as well as application of bibliometric methods to physics, chemistry, and technology. http://orcid.org/0000-0001-7025-7256

http://www.researcherid.com/rid/A-9925-2010

Max Planck Institute for Solid State Research Heisenbergstr., 1. 70569 Stuttgart, Germany r.haunschild@fkf.mpg.de

Lutz Bornmann works as a sociologist of science at the Division for Science and Innovation Studies in the Administrative Headquarters of the Max Planck Society in Munich (Germany). Since the late 1990s, he has been working on issues in the promotion of young academics and scientists in the sciences and on quality assurance in higher education. His current research interests include research evaluation, peer review, bibliometrics, and altmetrics. Thomson Reuters (http://highlycited. com) lists him among the most-highly cited researchers worldwide over the last ten years.

http://orcid.org/0000-0003-0810-7091

http://www.researcherid.com/rid/A-3926-2008

Division for Science and Innovation Studies Administrative Headquarters of the Max Planck Society Hofgartenstr., 8. 80539 Munich, Germany bornmann@gv.mpg.de

\section{Abstract}

Since the end of the 1980s, citation impact values -especially for evaluative purposes- are increasingly presented as fieldnormalized citation scores than as bare citation counts or citation rates. In rather popular variants of the scores, the average score over a publication year is not exactly one due to multiple Web of Science subject categories per paper. We propose a scaling method which introduces slight changes in the field-normalized scores of each paper that ensures that the average value of all scores equals one.

\section{Keywords}

Mean normalized citation score; Field normalized citation score; Scaling; Bibliometrics.

\section{Resumen}

Desde finales de la década de 1980, los valores de impacto de citación -especialmente para los propósitos de evaluación- se presentan cada vez más como valores de citación normalizada por disciplina más que simplemente como el número de citas o como porcentajes de citación. En las variantes más frecuentes de las puntuaciones, el promedio sobre un año de publicación no es exactamente igual a 1 debido a la posible asignación de un artículo a múltiples categorías temáticas de la Web of Science. Proponemos un método de escalado que introduce pequeños cambios en los valores de citación normalizada por disciplina de cada artículo, pero asegura que la media de todas las puntuaciones sea igual a uno.

\section{Palabras clave}

Citación media normalizada; Citación normalizada por disciplina; Escalado; Bibliometría. 
Haunschild, Robin; Bornmann, Lutz (2016). "Proposal of using scaling for calculating field-normalized citation scores". El profesional de la información, v. 25, n. 1, pp. 11-16.

http://dx.doi.org/10.3145/epi.2016.ene.02

\section{Introduction}

Since the end of the 1980 s, citation impact values - especially for evaluative purposes- are increasingly presented as field-normalized citation scores than as bare citation counts or citation rates (Schubert; Braun, 1986; 1993). For these scores, citation counts of a paper in question are compared with a corresponding reference set: the citation impact of papers published in the same year and subject category. Today, (1) journal sets from Web of Science (WoS, Thomson Reuters) or Scopus (Vinkler, 2010), (2) fields defined by citation relations between papers (Waltman; Van-Eck, 2012) and (3) field assignments by experts in a field (Bornmann; Daniel, 2008; Bornmann et al., 2011) are used as subject categories for normalization (journal sets, where many journals are assigned to multiple categories, have been used in most of the bibliometric studies). Only the normalization of the citation impact of a unit's (e.g. a university) publications with reference sets allows crossfield and cross-time comparisons between different units (e.g. universities with different disciplinary profiles and publishing in different time periods).

After a longer debate among bibliometricians on the use of averages of ratios and ratios of averages for the calculation of field-normalized citation scores (Bornmann; Mutz, 2011; Larivière; Gingras, 2011; Lundberg, 2007; Opthof; Leydesdorff, 2010; Van-Raan et al., 2010), the mean-normalized citation score (MNCS) has been proposed by Waltman, VanEck, Van-Leeuwen, Visser, and Van-Raan (2011) as a final solution. The MNCS employs averages of ratios (times cited over citations expected) with fractional counting of papers which are in multiple categories. The SCImago Institutions Ranking, InCites, and the Times Higher Education Ranking (THE Ranking) use another version of the MNCS which is also based on averages of ratios.

http://www.scimagoir.com/methodology.php

http://researchanalytics.thomsonreuters.com/m/pdfs/ indicators-handbook.pdf

https://www.timeshighereducation.co.uk/world-universityrankings/2015/world-ranking/\#tab-ranking-methodology

The difference is in the handling of papers which are in multiple subject categories. Here, the arithmetic average value of the normalized impact value in each category is used as the average impact for papers which are assigned to multiple categories (Rehn et al., 2014). The total impact of a collection of papers is calculated by the average value of the average values.

Today, the MNCS (in the different versions) can be seen as one of the most frequently used normalizing indicators in the field of (evaluative) bibliometrics (and thus can be seen as a standard in the field for impact normalization). The indicator is supposed to allow to decide whether a unit (scien- tist, institution, journal...) has published papers having produced an average citation impact $(M N C S=1)$ or an impact below $(M N C S<1)$ or above $(M N C S>1)$ the average in the field and publication year. These distinctions hold exactly for the MNCS if it is calculated via fractional (Waltman et al., 2011) or multiplicative (Herranz; Ruiz-Castillo, 2012) counting for papers which are assigned to multiple categories. In case of fractional counting, a paper's MNCS is based on fractionaIly calculated normalized impact values from different subject categories; in case of multiplicative counting, a paper's MNCS is considered multiple times depending on the subject categories the paper is assigned to. However, if the full counting method is used (as in the SCImago Institutions Ranking, the THE Ranking, and InCites), the average value over all MNCS values will differ slightly from one. In case of full counting, a paper's MNCS is calculated as a mean over the normalized impact values in the subject categories the paper is assigned to. The three counting methods will be illustrated in detail in section 2.

In this study, we would like to propose a small change in the calculation of the MNCS using the full counting approach. We will show that the calculation of the average value of all MNCS values for a publication year does not lead to exactly $M N C S=1$ in all cases, there are small deviations. Thus, we propose in the following to scale the MNCS using the mean MNCS over a publication year. Since our proposal can be simply considered in the calculation of MNCS values and leads to impact values which allow a better interpretation if the full counting method is used, we would like to bring it up for discussion in the bibliometric community.

\section{An example to illustrate the problem}

In the following, we consider the same example as in Waltman et al. (2011): Let us assume that the scientific universe consists of five publications where publications 1 and 2 belong to the scientific field $X$, publication 3 belongs to the field $Y$, publication 4 belongs to the field $Z$, and publication 5 belongs to the fields $X$ and $Y$, as shown in Table 1. The expected number of citations $(E)$ and normalized citation scores (NCS) using the fractional, multiplicative, and full counting approach are shown in Table 2.

Table 1. Hypothetical scientific universe with five publications, their assignments to scientific fields, and the citations they received

\begin{tabular}{|c|c|c|}
\hline Publication & Scientific field & Citations \\
\hline $\mathbf{1}$ & $\mathrm{X}$ & 2 \\
\hline $\mathbf{2}$ & $\mathrm{X}$ & 3 \\
\hline $\mathbf{3}$ & $\mathrm{Y}$ & 8 \\
\hline $\mathbf{4}$ & $\mathrm{Z}$ & 6 \\
\hline $\mathbf{5}$ & $\mathrm{X} \& \mathrm{Y}$ & 5 \\
\hline
\end{tabular}


Table 2. Expected citations (E) and normalized citations scores (NCS)

\begin{tabular}{|c|c|c|c|c|c|c|}
\hline Publication & $\boldsymbol{E}^{\text {frac }}$ & $\boldsymbol{E}^{\text {mult }}$ & $\boldsymbol{E}^{\text {full }}$ & $\boldsymbol{N C S}^{\text {frac }}$ & $\boldsymbol{N C S}^{\text {mult }}$ & $\boldsymbol{N C S}^{\text {full }}$ \\
\hline 1 & 3 & $10 / 3$ & $10 / 3$ & $2 / 3$ & $6 / 10$ & $6 / 10$ \\
\hline 2 & 3 & $10 / 3$ & $10 / 3$ & 1 & $9 / 10$ & $9 / 10$ \\
\hline 3 & 7 & $13 / 2$ & $13 / 2$ & $8 / 7$ & $16 / 13$ & $16 / 13$ \\
\hline 4 & 6 & 6 & 6 & 1 & 1 & 1 \\
\hline 5 & $21 / 5$ & $10 / 3 ; 13 / 2$ & $10 / 3 ; 13 / 2$ & $25 / 21$ & $15 / 10 ; 10 / 13$ & $590 / 520$ \\
\hline
\end{tabular}

It is straightforward to calculate the values of $E$ and NCS for publications 1-4 according to each counting method, because those four publications belong to a single scientific field. Publications 1 and 2 have the same value of $E$ because both belong to the scientific field $\mathrm{X}$ :

$$
\begin{gathered}
E_{1}^{\text {frac }}=E_{2}^{\text {frac }}=\frac{2+3+5 / 2}{1+1+1 / 2}=3 \\
E_{1}^{\text {mult }}=E_{2}^{\text {mult }}=E_{1}^{\text {full }}=E_{2}^{\text {full }}=\frac{2+3+5}{1+1+1}=\frac{10}{3}
\end{gathered}
$$

Publication 5 is counted half for the scientific fields $X$ and $Y$ each in the case of fractional counting, while it is counted fully in the cases of multiplicative and full counting. The NCS is simply the ratio of the number of observed citations and the number of expected citations: $N C S_{1}^{\text {frac }}=2 / 3, N C S_{2}$ frac $=$ $3 / 3=1$, and

$$
\begin{aligned}
& N C S_{1}^{\text {mult }}=N C S_{1}^{\text {full }}=\frac{2 / 1}{10 / 3}=\frac{6}{10} \\
& N C S_{2}^{\text {mult }}=N C S_{2}^{\text {full }}=\frac{3 / 1}{10 / 3}=\frac{9}{10}
\end{aligned}
$$

The NCS values of multiplicative and full counting agree with each other, but differ from the NCS value of fractional counting for publications 1 and 2 . This is due to the fact that publication 5 belongs to both fields $X$ and $Y$ which causes different $E$ values for fractional counting on one side and multiplicative as well as full counting on the other side.

Calculation of the $E$ value for publication 5 using fractional counting is done via the harmonic average of the $E$ values of scientific fields $\mathrm{X}$ and $\mathrm{Y}$ :

$$
E_{5}^{\text {frac }}=\frac{1+1}{1 / 3+1 / 7}=\frac{21}{5}
$$

In the multiplicative and full counting approaches, we have different $E$ values for publication 5 in scientific fields $X$ $\left[E_{5}{ }^{\text {mult }}(\mathrm{X})=E_{5}^{\text {full }}(\mathrm{X})=10 / 3\right]$ and $\mathrm{Y}\left[E_{5}{ }^{\text {mult }}(\mathrm{Y})=E_{5}^{\text {full }}(\mathrm{Y})=13 / 2\right]$, as publication 5 counts fully in both approaches. For calculation of the NCS value, the arithmetic average of the NCS values in scientific fields $X(15 / 10)$ and $Y(10 / 13)$ is used in the full counting approach, while publication 5 is counted twice in the multiplicative counting approach. The mean MNCS value over all five publications yields 1 for the fractional and multiplicative approaches, but differs slightly from one for the full counting approach:

$$
\begin{gathered}
M N C S_{\text {frac }}=\frac{\left(\frac{2}{3}+1+\frac{8}{7}+1+\frac{25}{21}\right)}{5}=1 \\
M N C S_{\text {mult }}=\frac{\left(\frac{6}{10}+\frac{9}{10}+\frac{16}{13}+1+\frac{15}{10}+\frac{10}{13}\right)}{6}=1 \\
M N C S_{\text {full }}=\frac{\left(\frac{6}{10}+\frac{9}{10}+\frac{16}{13}+1+\frac{590}{520}\right)}{5}=\frac{253}{260} \approx 0.973
\end{gathered}
$$

\section{Dataset}

The bibliometric data used in the following is from an inhouse database developed and maintained by the Max Planck Digital Library (MPDL, Munich) and derived from the Science Citation Index Expanded (SCI-E), Social Sciences Citation Index (SSCl), Arts and Humanities Citation Index $(\mathrm{AHCl})$ prepared by Thomson Reuters (Philadelphia, Pennsylvania, USA), Web of Science (WoS), as of May 15 2015.

\section{Results}

Table 3 shows average MNCS values (calculated on the basis of the full counting method) and average number of WoS categories for articles and reviews of the publication years 1980-2012. The MNCS shown in Table 3 was obtained by normalization with respect to the WoS subject categories and averaging over the impact values in each category for each paper. As papers can belong to multiple subject categories, the average MNCS on a higher aggregation level can differ from one, while the average MNCS over the WoS categories is still equal to one. The difference originates from papers which are assigned to multiple WoS subject categories because this introduces the average value of average values (see the example in section 2).

In fact, Table 3 shows that the MNCS deviates from one by $1 \%-4.2 \%$ in 24 of the 33 years shown in Table 3 . Therefore, the MNCS deviates by $1 \%$ or more in $73 \%$ of the years.

The average of all MNCS values per year is greater than one (negative deviation) only in 1993 and smaller than one (positive deviation) in the other years (cf. Table 3). The average number of WoS categories per paper has been increasing since 1980 as shown in Table 3. The relative increase of WoS categories per paper amounts to $22.5 \%$ (0.7\% per year on average). The maximum number of WoS subject categories per paper was 5 until 1985 and has increased to 6 in 1987. Also, the percentage of papers which are assigned to more than one subject category has increased from $25.8 \%$ in 1980 to $42.3 \%$ in 2012 . This is an increase of $63.9 \%$ (1.9\% per year). Both, increase of the average number of WoS ca- 
tegories per paper and the increase of the percentage of multiply categorized papers, increase the potential of a deviation of the MNCS from one over all MNCS values of a publication year.

The NCS for each paper averaged over the WoS categories $\left(N C S_{i}\right)$, where it has been assigned to, could be divided by the average value of all NCS values ( $\overline{M N C S}$ ) of a publication year:

$$
\begin{gathered}
\overline{M N C S}=\frac{1}{N} \sum_{i=1}^{N} N C S_{i} \\
N C I_{i}=\frac{N C S_{i}}{\overline{M N C S}}
\end{gathered}
$$

Here, $N$ is the number of papers in the publication year where this scaling is applied to, and $i$ indexes the papers. This scaling results in a slightly different impact value for each paper $\left(N \mathrm{Nl}_{i}\right)$ than before $\left(N C S_{i}\right)$ with the advantage that the average value of all $\mathrm{NCl}_{i}$ values equals one in each publication year and overall. This scaling approach will not alter the MNCS values of the fractional and multiplicative counting methods.

Returning to the example in Section 2, we have obtained MNCS $_{\text {frac }}=$ MNCS mult $_{\text {m }}=1$ and MNCS $_{\text {full }}=253 / 260 \neq 1$. The scaling approach proposed here would mean in this example that each $N C S_{i}^{\text {full }}$ value is multiplied with 260/253 in order to obtain the $\mathrm{NCl}_{i}^{\text {full }}$ values while the $\mathrm{NCS}_{i}^{\text {frac }}$ and $\mathrm{NCl}_{i}^{\text {frac }}$ values as well as the $\mathrm{NCS}_{i}^{\text {mult }}$ and $\mathrm{NCl}_{i}^{\text {mult }}$ values are equal:

$$
\begin{gathered}
N C I_{i}^{\text {frac }}=N C S_{i}^{\text {frac }} \\
N C I_{i}^{\text {mult }}=N C S_{i}^{\text {mult }} \\
N C I_{i}^{\text {full }}=N C S_{i}^{\text {full }} \frac{260}{253}
\end{gathered}
$$

This results in an average impact over all publications in this example of 1:

$$
M N C I_{\text {full }}=\frac{1}{5}\left(\frac{6}{10}+\frac{9}{10}+\frac{16}{13}+1+\frac{590}{520}\right) \frac{260}{253}=1
$$

\section{Discussion}

After demonstrating in this study that the MNCS does not lead to exactly $M N C S=1$ on the level of all papers within a publication year, we have proposed to scale each NCS value obtaining a slightly different $\mathrm{NCl}$ value for each individual paper to reach exactly an average $\mathrm{MNCl}$ value of 1 for each publication year. In the example presented in Section 2, this leads to an unusual situation: publication 4 is the only paper in its category, thereby having an NCS value of exactly 1 , but after our scaling the impact value of publication 4 is slightly larger than one: $260 / 253 \approx 1.03$. This can be considered as a disadvantage of the scaling approach, but this hypothetical example does not occur in practical bibliometrics. Since subject categories are based on journal sets or other large publication sets reflecting fields, reference sets generally contain more than one paper with different citation counts. Average citation counts over these subject categories usually do not lead to integers while citation counts of individual papers are always integer numbers. Therefore, the example of publication 4 is hypothetical and should not occur in bibliometric practice.

The NCS values of the full and multiplicative counting methods are the same for papers which belong to a single subject category. Differences in NCS values between both approaches are observed when papers are assigned to multiple subject categories. The scaling of NCS values using full counting affects all NCS values for each paper independent of the number of subject categories where the paper was assigned to. Therefore, the $\mathrm{NCl}$ values of the multiplicative and full counting approaches differ after scaling of the full counting NCS values. 
Although percentile-based approaches - especially the proportion of the $10 \%$ most frequently cited papers within a subject category and publication year - have been proposed as a more robust field normalizing method than MNCS (Hicks et al., 2015; Waltman et al., 2012), the MNCS is (still) widely used in evaluative bibliometrics. Thus, we would like to recommend scaling in the calculation of the MNCS. Also, a discussion might be necessary for other indicators when a paper is assigned to multiple subject categories (by using a full counting method). Other indicators might have the same problem as the MNCS

The proposal of scaling for the MNCS solves the same problem as the proposal of using the fractional counting method for calculating the MNCS. This method is similar to the proposal of using the fractional counting method for calculating the proportion of the $10 \%$ most frequently cited papers within a subject category and publication year. Starting from the observations that one does not receive exactly $10 \%$ for the $10 \%$ most frequently cited papers within a subject category and publication year (but deviations of around 1\% within certain disciplines), Waltman and Schreiber (2013) proposed a fractional counting method. Here, publications at the threshold of $10 \%$ are fractionally counted as top $10 \%$ papers or below $90 \%$ papers. This approach ensures (similar to scaling for the MNCS) that one has exactly $10 \%$ top $10 \%$ papers. The fractional counting approach is used for the Leiden Ranking (Waltman et al., 2012).

Although MNCS (and NCS) values should not be interpreted to the precision provided in tables (Hicks et al., 2015), it is very useful if the average of all NCS values (MNCS) of a publication year equals exactly one.

\section{Acknowledgements}

The bibliometric data used in this paper are from an inhouse database developed and maintained by the Max Planck Digital Library (MPDL, Munich) and derived from the Science Citation Index Expanded (SCI-E), Social Sciences Citation Index (SSCl), Arts and Humanities Citation Index ( $\mathrm{AHCl}$ ) prepared by Thomson Reuters (Philadelphia, Pennsylvania, USA). We thank Ludo Waltman for helpful comments on a previous version of the manuscript.

\section{Bibliography}

Bornmann, Lutz; Daniel, Hans-Dieter (2008). "Selecting manuscripts for a high impact journal through peer review: a citation analysis of Communications that were accepted by Angewandte Chemie International Edition, or rejected but published elsewhere". Journal of the American Society for Information Science and Technology, v. 59, n. 11, pp. 1841-1852.

http://dx.doi.org/10.1002/asi.20901

Bornmann, Lutz; Mutz, Rüdiger (2011). "Further steps towards an ideal method of measuring citation performance: the avoidance of citation (ratio) averages in field-normalization". Journal of informetrics, v. 5, n. 1, pp. 228-230. http://www.lutz-bornmann.de/icons/AverageValue.pdf http://dx.doi.org/10.1016/j.joi.2010.10.009

Bornmann, Lutz; Schier, Hermann; Marx, Werner; Daniel,
Hans-Dieter (2011). "Is interactive open access publishing able to identify high-impact submissions? A study on the predictive validity of Atmospheric Chemistry and Physics by using percentile rank classes". Journal of the American Society for Information Science and Technology, v. 62, n. 1, pp. 61-71.

http://dx.doi.org/10.1002/asi.21418

Herranz, Neus; Ruiz-Castillo, Javier (2012). "Sub-field normalization in the multiplicative case: Average-based citation indicators". Journal of informetrics, v. 6, n. 4, pp. 543-556.

http://dx.doi.org/10.1016/j.joi.2012.02.006

Hicks, Diana; Wouters, Paul; Waltman, Ludo; De-Rijcke, Sarah; Rafols, Ismael (2015). "Bibliometrics: The Leiden Manifesto for research metrics". Nature, v. 520, n. 7548, pp. 429-431.

http://www.nature.com/news/bibliometrics-the-leidenmanifesto-for-research-metrics-1.17351

Larivière, Vincent; Gingras, Yves (2011). “Averages of ratios vs. ratios of averages: an empirical analysis of four levels of aggregation". Journal of informetrics, v. 5, n. 3, pp. 392-399.

http://dx.doi.org/10.1016/j.joi.2011.02.001

Lundberg, Jonas (2007). "Lifting the crown-citation z-score". Journal of informetrics, v. 1, n. 2, pp. 145-154.

http://dx.doi.org/10.1016/j.joi.2006.09.007

Opthof, Tobias; Leydesdorff, Loet (2010). "Caveats for the journal and field normalizations in the CWTS ('Leiden') evaluations of research performance". Journal of informetrics, v. 4, n. 3, pp. 423-430.

http://dx.doi.org/10.1016/j.joi.2010.02.003

Rehn, Catharina; Wadskog, Daniel; Gornitzki, Carl; Larsson, Agne (2014). Bibliometric indicators - Definitions and usage at Karolinska Institutet. Karolinska Institutet.

http://kib.ki.se/sites/default/files/bildarkiv/Dokument/ bibliometric_indicators_2014.pdf

Schubert, Andras; Braun, Tibor (1986). "Relative indicators and relational charts for comparative assessment of publication output and citation impact". Scientometrics, v. 9, n. 5-6, pp. 281-291.

http://dx.doi.org/10.1007/BF02017249

Schubert, Andras; Braun, Tibor (1993). "Reference standards for citation based assessments". Scientometrics, v. 26, n. 1, pp. 21-35.

http://dx.doi.org/10.1007/BF02016790

Van-Raan, Anthony F. J.; Van-Leeuwen, Ted N.; Visser, Martin S.; Van-Eck, Nees J.; Waltman, Ludo (2010). "Rivals for the crown: reply to Opthof and Leydesdorff". Journal of informetrics, v. 4, n. 3, pp. 431-435.

http://arxiv.org/pdf/1003.2113.pdf

http://dx.doi.org/10.1016/j.joi.2010.03.008

Vinkler, Peter (2010). The evaluation of research by scientometric indicators. Oxford, UK: Chandos Publishing. ISBN: 9781843345725

Waltman, Ludo; Calero-Medina, Clara; Kosten, Joost; No- 
yons, Ed C. M.; Tijssen, Robert J. W.; Van-Eck, Nees J.; VanLeeuwen, Thed N.; Van-Raan, Anthony F. J.; Visser, Martijn, S.; Wouters, Paul (2012). "The Leiden Ranking 2011/2012: data collection, indicators, and interpretation". Journal of the American Society for Information Science and Technology, v. 63, n. 12, pp. 2419-2432.

http://arxiv.org/pdf/1202.3941.pdf

http://dx.doi.org/10.1002/asi.22708

Waltman, Ludo; Schreiber, Michael (2013). "On the calculation of percentile-based bibliometric indicators". Journal of the American Society for Information Science and Technology, v. 64, n. 2, pp. 372-379.

http://arxiv.org/pdf/1205.0646.pdf http://dx.doi.org/10.1002/asi.22775

Waltman, Ludo; Van-Eck, Nees J. (2012). "A new methodology for constructing a publication-level classification system of science". Journal of the American Society for Information Science and Technology, v. 63, n. 12, pp. 2378-2392. http://arxiv.org/pdf/1203.0532.pdf http://dx.doi.org/10.1002/asi.22748

Waltman, Ludo; Van-Eck, Nees J.; Van-Leeuwen, Thed N.; Visser, Martin S.; Van-Raan, Anthony F. J. (2011). "Towards a new crown indicator: some theoretical considerations". Journal of informetrics, v. 5, n. 1, pp. 37-47. http://arxiv.org/pdf/1003.2167.pdf http://dx.doi.org/10.1016/j.joi.2010.08.001

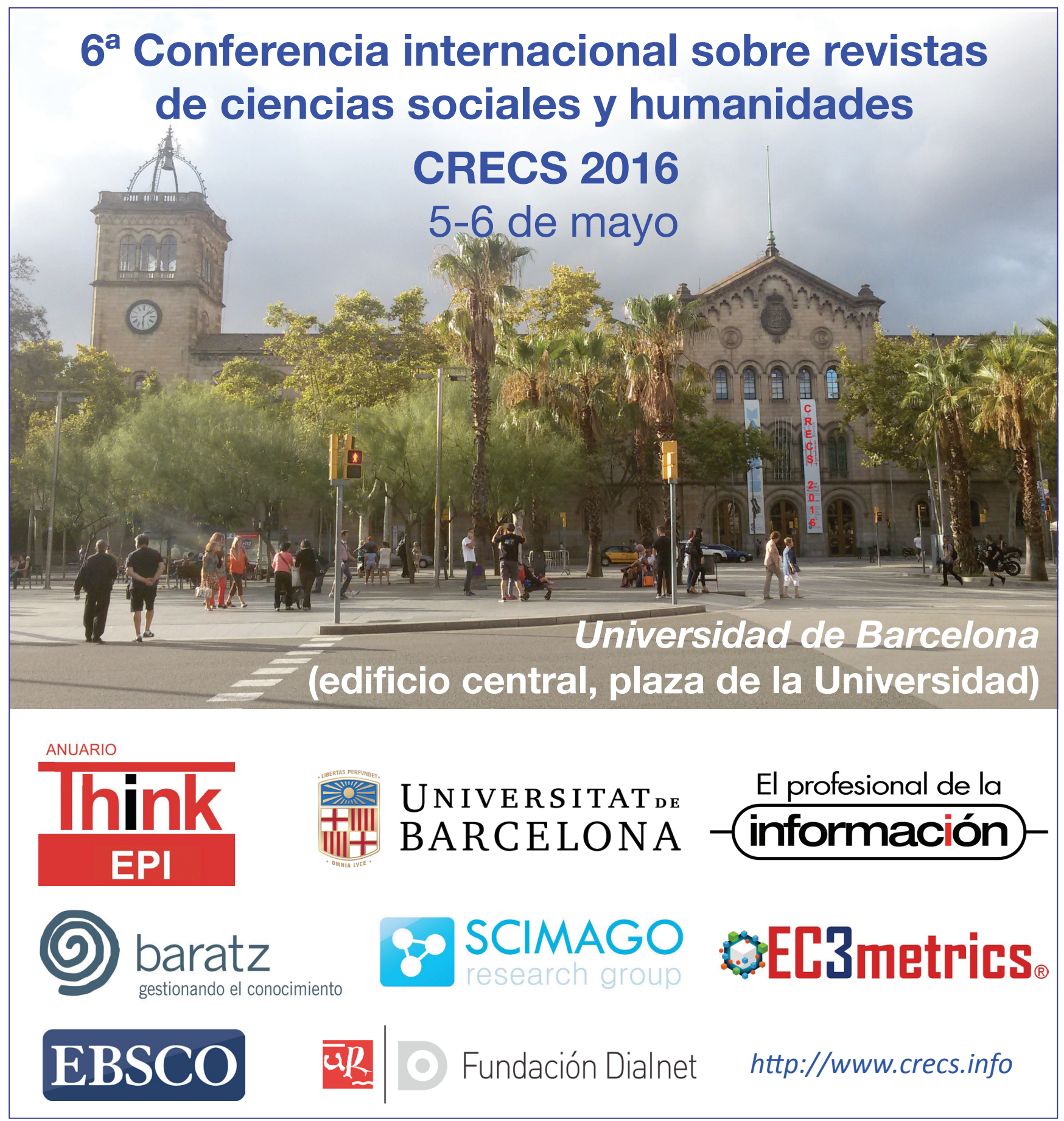

